\title{
Evaluating IBD Patient-Initiated Electronic Communication During the SARS-CoV-2 Pandemic: A Single-Center Study
}

\author{
Sanjana Rao ${ }^{1}$ Marie L. Borum ${ }^{1}$ \\ Received: 17 March 2021 / Accepted: 26 March 2021 / Published online: 21 May 2021 \\ (c) The Author(s), under exclusive licence to Springer Science+Business Media, LLC, part of Springer Nature 2021
}

\section{To the Editor,}

It is with great interest that we read the article by Perisetti and Goyal, entitled "Successful Distancing: Telemedicine in Gastroenterology and Hepatology during the COVID-19 Pandemic," which discussed the importance of integrating telemedicine platforms with gastroenterology care in the context of limited interactions between inflammatory bowel disease (IBD) patients and their care providers [1]. While the paper discussed an increase in IBD patients' use of telemedicine modalities, such as online applications, it also shed light on barriers to electronic communication during this vulnerable time, citing low acceptability of telemedicine use among Black and elderly patients. We would like to further elaborate on these findings with our own single-center study regarding demographic patterns in portal messaging by IBD patients during the SARS-CoV-2 outbreak.

Through our retrospective chart review, IBD patients were seen within the past 3 years by gastroenterologists at our institution. We recorded patient gender, age, race, IBD subtype and IBD treatment regimen. E-messaging use and content via the secure FollowMyHealth portal application between March 1, 2020 and August 31, 2020 were evaluated. Statistical analyses were conducted using a two-tailed Fisher's Exact test with significance set at $p<0.05$.

A total of 295 medical records of IBD patients were reviewed. There were 130 (44.1\%) males and 165 (55.9\%) females, with a mean age of 45.7 years. Seventy-six (25.8\%) had Crohn's disease (CD) and 208 (70.4\%) had ulcerative

Marie L. Borum

mborum@mfa.gwu.edu

Sanjana Rao

sanjanarao@gwmail.gwu.edu

1 The Division of Gastroenterology and Liver Diseases, George Washington University Medical Center, 2150 Pennsylvania Avenue, NW, Suite 3-405, Washington, D.C. 20037, USA colitis (UC). During the outbreak, 53 (18.0\%) of the IBD patients engaged in secure e-communication with their gastroenterologist. Patients on non-biologic therapy contacted providers significantly less than those on biologic medication $(54.7 \%$ vs. $45.3 \%$; $\mathrm{p}<0.001)$. The most common messages included concerns regarding SARS-CoV-2 risk given their immunosuppressive medication (18.9\%), home infusion arrangements to avoid exposure (15.1\%), and refill requests (18.9\%). Electronic communication was initiated significantly more by males than females $(56.6 \%$ vs. $43.4 \% ; p=0.0478)$ and White patients than AfricanAmerican patients (71.7\% vs. $17.0 \%, p=0.0109)$. Lastly, there was no significant difference in the use of e-messaging between patients $\leq 50$ years of age and those $>50(66.0 \%$ vs. $34.0 \% ; p=1)$, nor IBD subtype (79.3\% UC vs. $15.1 \% \mathrm{CD}$; $p=0.0774)$.

In our biopsychosocial analysis of e-messaging use by our IBD cohort, safety concerns dominated patients' engagement in portal messaging with their providers given their potential immunological fragility, as well as the similarities between COVID-19 and IBD symptomology. In the context of welldocumented mistrust of physicians among racial minorities, our findings of African-Americans using portal messaging significantly less than other patient populations warrant further exploration and intervention to ensure equity of care among all ethnic groups. In contrast to the article presented, there was no discrepancy in e-communication engagement by age group. This finding is encouraging in the setting of a shift toward greater utilization of virtual communication and may speak to the incentivization of older individuals to receive care while minimizing risk of disease transmission. Ultimately, further research is warranted to define and rectify the obstacles and facilitators in telehealth use such that providers can offer optimal, equitable care. 


\section{Reply}

We thank Rao S and Borum ML for their interest in our article "Successful Distancing: Telemedicine in Gastroenterology and Hepatology during the COVID-19 Pandemic [1]." In their correspondence, the authors provide their findings on using electronic communication patterns via portal messaging in inflammatory bowel disease (IBD) patients during the COVID-19 pandemic.

COVID-19 has posed numerous unique challenges and dilemmas for managing IBD patients, especially due to similarities between COVID-19 intestinal symptoms and IBD flare $[2,3]$. The use of telemedicine among IBD patients has been on the rise during the pandemic. The authors showed significantly low use of e-communication among AfricanAmericans (17\%) compared to White (71.7\%) patients with IBD. These findings are in concordance with other studies on telemedicine use [1]. Interestingly, Rao et al. study also finds that patients' age and IBD subtype (Crohn's or ulcerative colitis) did not predict the electronic communication response. The use of telemedicine for IBD can be cost-saving and effective in improving quality indicators and decreasing hospitalization rates [4]. Telemedicine use has been further extended to be used in pediatric IBD patients with acceptable results [4]. However, the performance status of disease activity indexes in the telemedicine is still not clear, highlighting the need for standardization of the tools.

Another area of significance on this topic is an element of "trust" in telemedicine in IBD patients recently reported by Costantino et al. during the pandemic [5]. In their study, more than $75 \%$ reported increased trust in telemedicine for their IBD care. Regardless, even gastroenterologists are feeling more comfortable than ever for telemedicine use. Although increased telemedicine use is key to expanding IBD care, the importance of racial differences noted in this letter by Rao et al. is vital to identify the potential barriers, especially among minorities. These could be potential targets for public health intervention for future studies.

Telemedicine in IBD: A COVID Conundrum

Abhilash Perisetti, Hemant Goyal

Abhilash Perisetti, MD FACP

Department of Gastroenterology and Hepatology, University of Arkansas for Medical Sciences, Little Rock, AR, USA.

Email: abhilash.perisetti@gmail.com
Hemant Goyal, MD FACP

${ }^{1}$ The Wright Center for Graduate Medical Education, 501

S. Washington Avenue, Scranton, PA 18503 USA.

Office: 570.591 .5175

Email: doc.hemant@yahoo.com

${ }^{2}$ Assistant Clinical Professor of Medicine, Mercer University School of Medicine, 707 Pine St., Macon, GA 31201 USA.

Author's contribution Conception and design: Hemant Goyal, Abhilash Perisetti, Literature review: Abhilash Perisetti, Hemant Goyal, First Draft: Abhilash Perisetti, Critical revision and editing: Hemant Goyal, Final approval: Hemant Goyal, Abhilash Perisetti.

\section{Declarations}

Conflict of interest Hemant Goyal held stocks in Conversion Labs, Inc.

\section{Reference}

1. Perisetti A, Goyal H. Successful Distancing: Telemedicine in Gastroenterology and Hepatology During the COVID-19 Pandemic. Dig Dis Sci. 2021;3:1-9. https://doi.org/10.1007/ s10620-021-06874-x.

2. D'Amico F, Danese S, Peyrin-Biroulet L. Systematic Review on Inflammatory Bowel Disease Patients With Coronavirus Disease 2019: It Is Time to Take Stock. Clin Gastroenterol Hepatol. 2020;18:2689-700.

3. Gajendran M, Perisetti A, Aziz M, Raghavapuram S, Bansal P, Tharian B, et al. Inflammatory bowel disease amid the COVID-19 pandemic: impact, management strategies, and lessons learned. Ann Gastroenterol. 2020;33:591-602.

4. de Jong MJ, Boonen A, van der Meulen-de Jong AE, RombergCamps MJ, van Bodegraven AA, Mahmmod N, et al. Cost-effectiveness of telemedicine-directed specialized vs standard care for patients with inflammatory bowel diseases in a randomized trial. Clin Gastroenterol Hepatol. 2020;18:1744-52.

5. Costantino A, Noviello D, Mazza S, Berté R, Caprioli F, Vecchi M. Trust in telemedicine from IBD outpatients during the COVID-19 pandemic. Dig Liver Dis. 2021;53:291-94.

Publisher's Note Springer Nature remains neutral with regard to jurisdictional claims in published maps and institutional affiliations. 